\title{
Diffusion Smoothing on the Cortical Surface
}

\author{
Moo K. Chung ${ }^{*}$, Keith J. Worsley ${ }^{* \dagger}$, Jonathan Taylor ${ }^{*}$, Jim Ramsay $^{\ddagger}$, Steve Robbins ${ }^{\dagger}$, Alan C. Evans ${ }^{\dagger}$ \\ "Department of Mathematics and Statistics \\ ${ }^{\top}$ Montreal Neurological Institute \\ 'Department of Psychology, McGill University
}

\section{Abstract}

Gaussian kernel smoothing has been widely used in either 2D flat or 3D volume images, but it does not work on the curved cortical surface. However, by reformulating Gaussian kernel smoothing as a solution to a diffusion equation on a 2D manifold, we can generalize it to the cortical surface. This generalization is called diffusion smoothing and has been used in analysis of fMRI data on the cortical surface [1] and detecting cortical surface-area growth [3]. We give an exact mathematical formulation for the diffusion smoothing on triangulated cortical surfaces so that this technique can be used for any surface-based functional and structural analysis. As an illustration, we smooth the mean curvatures on the outer cortical surfaces to show how the the smoothing actually incorporates the geodesic curvature information of the surface.

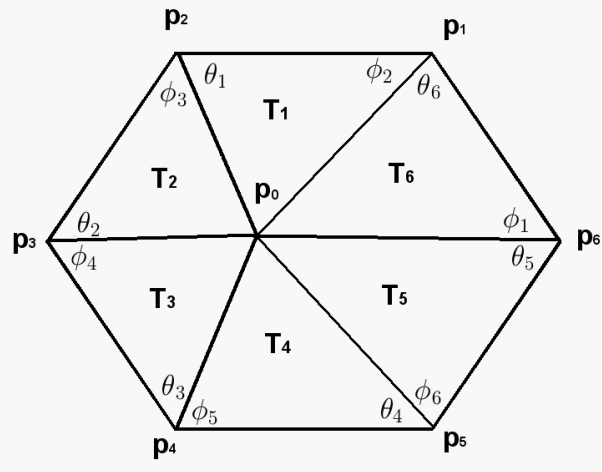

Figure 1: A typical triangulation

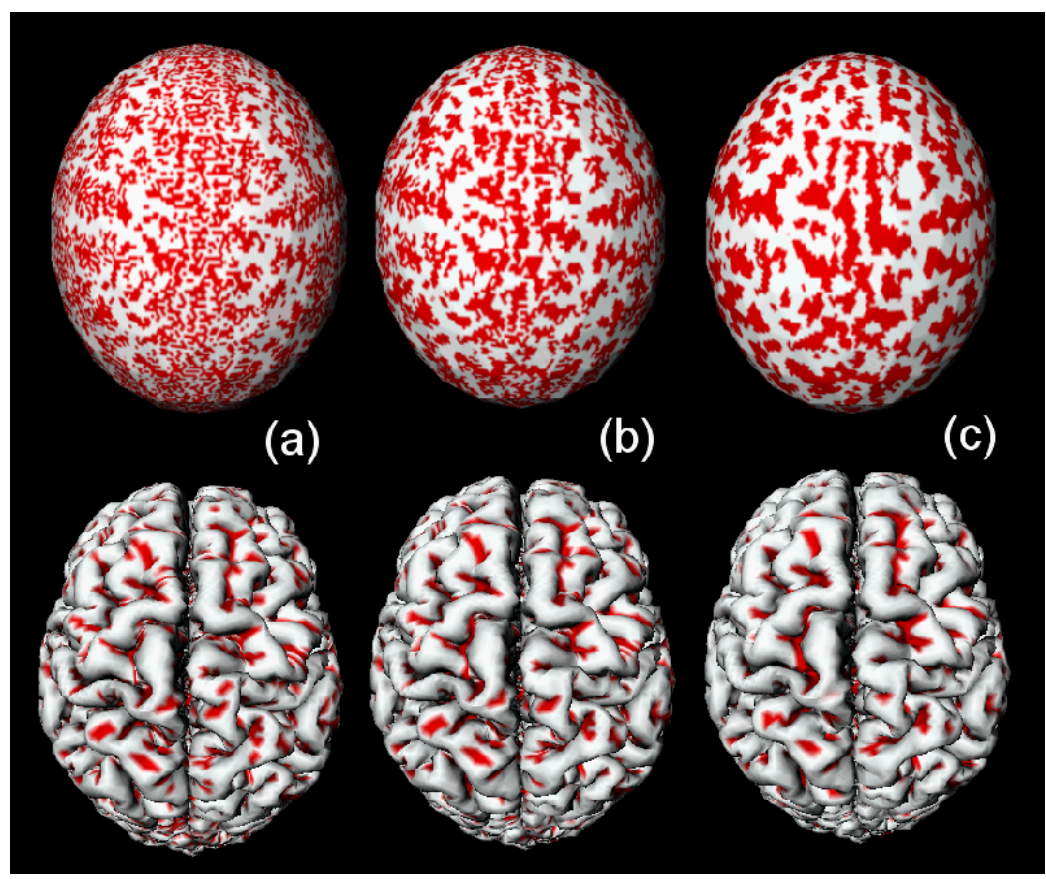

Figure 2: The mean curvature from the outer cortex is mapped onto an ellipsoid during the diffusion smoothing. (a) Before the iteration (b) After 40 iterations (c) After 100 iterations

\section{Methods}

Gaussian kernel smoothing of the signal $f(x)$ in n-dimension with FWHM=4( $\ln 2)^{1 / 2} t^{1 / 2}$ is defined as the convolution of the n-dimensional Gaussian kernel $G(x ; t)$ with the signal $f(x)$, i.e. $F(x, t)=f^{*} G(x ; t)$. It can be shown that the convoluted signal $F$ is the solution of a diffusion equation $d F / d t=L[F]$ with the n-dimensional Euclidean Laplacian $L$. Since the cortical surface in non-Euclidean, the Euclidean Laplacian is not well defined on the cortical surface. The generalization of the Laplace operator $L$ to an arbitrary curved surface is called the Laplace-Beltrami operator and it is defined in terms of the Riemmanian metric tensors.

In order to estimate the Laplace-Beltrami operator on a triangulated cortical surface, we have used the finite element method [2]. Let $F\left(p_{i}\right)$ be the signal on the $i$-th node $p_{i}$ in the triangulation. If $p_{1}, \ldots, p_{m}$ are $m$-neighboring nodes around $p=p_{0}$, the LaplaceBeltrami operator at $p$ is estimated by 
$L[F(p)]=w_{1}\left(F\left(p_{1}\right)-F(p)\right)+\ldots+w_{m}\left(F\left(p_{m}\right)-F(p)\right)$

with the weights $w_{i}=\left(\cot \phi_{i}+\cot \theta_{i}\right) /\left(T_{1}+\ldots+T_{m}\right)$, where $\phi_{i}$ and $\theta_{i}$ are the two angles opposite to the edge $p_{i}-p$ in triangles and $T_{1}+\ldots+T_{m}$ is the sum of the areas of $m$-incident triangles at $p$ (Figure 1). Then the diffusion equation is solved via the finite difference scheme:

$F\left(p_{i}, t_{n+1}\right)=F\left(p_{i}, t_{n}\right)+\left(t_{n+1}-t_{n}\right) L\left[F\left(p_{i}, t_{n}\right)\right]$ with the initial condition $F\left(p_{i}, t_{0}\right)=f\left(p_{i}\right)$. After $N$-iterations, the diffused signal is locally equivalent to the Gaussian kernel smoothing with FWHM=4(ln2) ${ }^{1 / 2} N^{1 / 2}\left(t_{n}-t_{0}\right)^{1 / 2}[2]$.

\section{Results}

The ASP method [3] is used to extract the outer cortical surfaces each consisting of 81920 triangles from MR scans. At this surface sampling rate, the average intervertex distance is about $4 \mathrm{~mm}$. The mean curvature $f\left(p_{i}\right)$ of the cortical surface is computed based on the least-squares estimation of a local quadratic surface [2]. Figure 2 shows the diffusion smoothing of the mean curvature with $5 \mathrm{~mm}$ FWHM. If the smoothing were based on simple inter-nodal averaging, such sulcal pattern is not possible to obtain.

\section{Conclusion}

Gaussian kernel smoothing can be generalized to cortical surfaces enabling surface-based statistical analysis. The numerical implementation will be freely available as Matlab code on the web at http://www.math.mcgill.ca/keith/BICstat.

\section{References}

[1] Andrade A et al., Detection of fMRI Activation on the Cortical Surface, NeuroImage, 2000 (in press).

[2] Chung MK et al., http://www.math.mcgill.ca/chung/diffusion/diffusion.pdf, 2000

[3] Chung MK et al., Statistical Analysis of Cortical Surface Area Change, with an Application to Brain Growth, HBM2001

Conference

[4] MacDonald D et al., NeuroImage 12:340-356, 2000 